\title{
Cumplimiento de la NOM 253-SSA1-2012, para la administración de hemoderivados en un hospital de segundo nivel
}

\author{
Compliance with NOM 253-SSA1-2012, for the administration \\ of hemoderives in a second-level hospital
}

\author{
Elsa Álvarez Bolaños, ${ }^{*}$ Martha Venegas Rivas, ${ }^{\ddagger}$ Oscar Geovanny Granados Pérez, \\ Miguel Ángel Cuevas-Budhart?
}

\begin{abstract}
RESUMEN
Introducción: La seguridad de las transfusiones se reconoce como una prioridad global; en México se cuenta con la NOM-253-SSA1-2012, para la disposición de sangre humana y sus componentes con fines terapéuticos; sin embargo, presenta desviaciones en su cumplimiento, respecto al indicador de calidad para la atención de enfermería en el IMSS, "procedimiento de transfusiones sanguíneas», por lo cual se hace un tema prioritario para los gerentes y profesionales. Objetivo: Evaluar el nivel de cumplimiento a lo establecido por la NOM 253-SSA1-2012 administración de hemoderivados en la atención de enfermería del Hospital General Regional No. 1 «Vicente Guerrero». Material y métodos: Estudio observacional, retrospectivo, transversal y descriptivo, con población de 24,470 eventos transfusionales, se realizó un muestreo aleatorizado simple, con un tamaño de muestra de 237 pacientes transfundidos por enfermería en el Hospital General Regional No. 1 «Vicente Guerrero». Para la recogida de información se utilizó cédula de evaluación del indicador de calidad de atención de enfermería; «transfusión de hemoderivados» 253-SSA1-2012 (cinco ítems). Resultados: El porcentaje general de cumplimiento del indicador de calidad fue 51.8\% por parte de Enfermería; mientras que por parte del Banco de Sangre fue de $9.87 \%$. El criterio con mayor cumplimiento fue «registro de cantidad de unidades, volumen, número de identificación de las unidades de sangre o complemento» (94.44\%), el criterio con menor desempeño fue «control de signos vitales antes, durante y después de la transfusión» (67.95\%). Conclusión: Los resultados muestran un incumplimiento elevado del indicador de calidad para la administración de hemoderivados, lo cual pone en riesgo la calidad del proceso de transfusión y por ende la seguridad del paciente.
\end{abstract}

Palabras clave: Atención de enfermería, calidad asistencial, hemoderivados, indicador de resultado, indicadores de calidad, seguridad del paciente.

\footnotetext{
ABSTRACT

Introduction: The safety of transfusions is recognized as a global priority; in Mexico there is NOM-253SSA1-2012 for the disposal of human blood and its components for therapeutic purposes, however, this quality indicator for nursing care shows deviations in compliance, which is why a priority issue for managers and professionals. Objetive: To evaluate the level of compliance with the provisions of NOM 253-SSA1-2012 administration of blood products in the Regional General Hospital No. 1 «Vicente Guerrero». Material and methods: Observational, retrospective, cross-sectional and descriptive study, with a population of 24,470 transfusion events, a simple randomized sample was carried out, with a sample size of 237 patients transfused by nursing in the Regional Hospital No. 1 «Vicente Guerrero». For the collection of information, the nursing care quality indicator was used; «transfusion of blood products» 253-SSA1-2012 (5 items). Results: The general percentage of compliance with the quality indicator was $51.8 \%$, the criterion with the highest compliance «record of number of units, volume,
}

* Coordinación

Delegacional de

Enfermería en Atención Médica, IMSS Guerrero, México.

₹ Banco de Sangre del Hospital General Regional No. 1 «Vicente Guerrero», IMSS. Acapulco, Guerrero, México.

${ }^{\S}$ Pasante de Servicio Social en Enfermería. "Coordinador de Programas de Enfermería. Unidad de Investigación Médica en Enfermedades Nefrológicas, CMN Siglo XXI.

Correspondencia: MÁCB, angel_budhart@hotmail. com/migcueva@ucm.es Conflicto de intereses: Ninguno.

Citar como: Álvarez BE, Venegas RM, Granados POG, Cuevas-Budhart MÁ. Cumplimiento de la NOM 253-SSAT-2012, para la administración de hemoderivados en un hospital de segundo nivel. Rev CONAMED. 2021; 26(1): 35-41. https://dx.doi. org/10.35366/99126 Financiamiento: Ninguno.

Recibido: 17/07/2019. Aceptado: 05/03/2021. 
identification number of blood units or complement» (94.44\%), the criteria with the lowest performance was «control of vital signs before, during and after the transfusion» (67.95\%). Conclusion: The results show a high non-compliance of the quality indicator for the blood products administration, which puts the quality of the transfusion process at risk and therefore the patient's safety.

Keywords: Blood products, nursing care, patient safety, quality indicators, quality of care, result indicator.

\section{INTRODUCCIÓN}

Proporcionar una atención de calidad es un tema prioritario para los gerentes y profesionales sanitarios, ${ }^{1,2}$ por lo que se han diseñado programas estratégicos para garantizar el bienestar del paciente, como las acciones esenciales de seguridad del paciente diseñados por la Secretaría de Salud para regular procedimientos con estándares de calidad, ${ }^{3}$ los cuales sirven para objetivar aspectos relevantes de la asistencia, establecer comparaciones, proponer objetivos y crear una cultura de evaluación y mejora de la calidad de la atención médica.4-6

La Organización Mundial de la Salud (OMS) y la Organización Panamericana de la Salud (OPS) establecen que para abastecer de sangre segura a la población se debe fomentar el trabajo en equipo, obtener la sangre y componentes sanguíneos de donantes voluntarios y altruistas, no remunerados y regulares, asegurándose de que reciban una atención de calidad.?

Ante ello, el Gobierno de México cuenta con la Norma Oficial Mexicana NOM-253-SSA1-2012,? para la disposición de sangre humana y sus componentes con fines terapéuticos, con el fin de garantizar la autoeficacia, cobertura universal y seguridad de la sangre y sus componentes, la cual fue creada hace más de 20 años desde que fue publicada la primer NOM por la Secretaría de Salud; sin embargo, existen desviaciones normativas en su cumplimiento en el procedimiento de transfusiones sanguíneas. ${ }^{8}$

Cabe señalar que la transfusión sanguínea es una técnica básicamente de enfermería que requiere un conocimiento profundo de las bases fisiológicas y un manejo meticuloso de la atención al paciente y la aplicación correcta de un protocolo, pero fundamentalmente prevenir las serias complicaciones que pueden presentarse para evitar errores en la selección y administración de los productos, prevenir y controlar posibles complicaciones, realización de la técnica de forma sistemática con una valoración del paciente durante la realización de la técnica de administración de hemoderivados., 90

Para los profesionales de enfermería, la transfusión de hemoderivados forma parte de la práctica clínica diaria, tomando en cuenta todos los avances científicos y tecnológicos en el campo de la medicina transfusional y la rigurosa normativa en materia de seguridad transfusional, sigue persistiendo el riesgo de provocar efectos adversos relacionados con la transfusión que, en ocasiones, pueden ser fatales para el paciente.1,12

De acuerdo con esta problemática, diversos estudios se han realizado en este contexto, como el de Morales-Olarte E y colaboradores, ${ }^{13}$ donde identificaron que los problemas de enfermería durante la transfusión de componentes sanguíneos fueron principalmente por el subregistro en aproximadamente $50 \%$ de los casos en los tres turnos, independientemente del componente transfundido.

Por otro lado, Flores-Torrecillas R y su equipo ${ }^{14}$ determinaron una eficacia global de «parcial» a «excelente» logrando 65.4\%, la evaluación del desempeño incluye los puntos obligatorios de la NOM003-SSA2-1993 de Transfusión de hemoderivados: las enfermeras revisan los datos de los pacientes en la solicitud de productos sanguíneos el 99.1\%, coteja nuevamente los datos del paciente y del hemoderivado 60.9\%. El 90.9\% del personal de enfermería registra signos vitales y el $22.7 \%$ inicia la transfusión y permanece con el paciente los primeros 15 minutos. El 99.1\% registra signos vitales al suspenderse la transfusión por complicaciones y ninguno tomó muestra sanguínea.

Velasco-Rodríguez R y colaboradores ${ }^{15}$ identificaron un incumplimiento en observación y revisaron el estado de la piel 60.4\%, lavado de manos $52 \%$, no registraron los signos vitales después de la transfusión 29.6\%, no los tomaron ni los registraron antes del procedimiento 18.7\%. Con relación al nivel de cumplimiento, $75 \%$ de enfermeras cumple el manejo de hemoderivados con un nivel de mínimo a excelente, y predomina el cumplimiento parcial con $37.5 \%$. 
Como se puede observar, son diversos los resultados ante esta problemática, lo que no permite evidenciar un comportamiento unánime a nivel nacional, por lo cual es insuficiente la información y la producción científica sobre este contexto.

Por lo anterior, es primordial comprender la situación del cumplimiento de los indicadores de calidad en relación con la «NOM-253-SSA1-2012, para la disposición de sangre humana y sus componentes con fines terapéuticos», ${ }^{7}$ se planteó el objetivo de evaluar el nivel de cumplimiento a lo establecido por dicha norma, administración de hemoderivados en la atención de enfermería del Hospital General Regional No. 1 «Vicente Guerrero».

\section{MATERIAL Y MÉTODOS}

Estudio observacional, descriptivo, retrospectivo para los datos de «registro del proceso de transfusión» del procedimiento normativo institucional, y la fase transversal para evaluar la atención de enfermería de acuerdo con la NOM-253-SSA1-2012 «transfusión de hemoderivados». Se realizó un muestreo probabilístico aleatorizado simple con un intervalo de confianza del 95\%, una precisión del $3 \%$ y una proporción del 5\%. Se ajustó a las pérdidas un 15\%, con lo cual quedó constituido un tamaño de muestra de 237 pacientes transfundidos por enfermería de 24,470 eventos transfusionales entre enero del 2016 y abril del 2018 en el Hospital General Regional No. 1 «Vicente Guerrero».

Para efectos del análisis y evaluación de la calidad se utiliza el enfoque sistémico diferenciando tres áreas, a saber: la estructura, los procesos y los resultados. Por lo cual, los indicadores de calidad que se evaluaron fueron de resultados, ${ }^{16}$ siendo éstos registro del proceso de trasfusión y procedimiento de transfusiones sanguíneas.

Instrumentos de medición: para la recolección de información se utilizaron dos instrumentos, para medir el cumplimiento se utilizó la cédula de evaluación del indicador de calidad de atención de enfermería del IMSS NOM-253-SSA1-2012 «transfusión de hemoderivados» validado por la División de Mejora a la Gestión de los Servicios de Salud (DMCSS) $)^{17}$ y la Unidad de Evaluación de Delegaciones (UED), constituido con cinco ítems: 1) cantidad de unidades, volumen, número de identificación de las unidades de sangre o complemento;
2) registro de fecha y hora de inicio y término de la transfusión; 3) control de signos vitales y estado general del paciente antes, durante y después de la transfusión; 4) en caso de reacciones adversas a la transfusión se registra su tipo y los procedimientos realizados; 5) nombre completo y firma del médico que indicó la transfusión, así como el personal de salud responsable de la transfusión.

También se tomó en cuenta para la evaluación el documento normativo de registro de «procedimiento para el soporte transfusional» IMSS 2430021-026 que consta de cinco a partados: 1) datos del paciente; 2) datos de la unidad(es) transfundida(s); 3) datos de la(s) transfusión(es); 4) resumen de la(s) transfusión(es); 5) observaciones. Este documento evalúa el área médica de banco de sangre, donde se coteja con orden médica y solicitud de transfusión de los cuales se evaluó el cumplimiento del indicador de transfusión sanguínea de acuerdo con lo señalado en la NOM 253-SSA1-2012.

Ambos instrumentos se apegan a lo que establece la NOM 253-SSA1-2012 en el apartado 19 sobre «procedimientos normalizados de operación, guías, instructivos, documentos y registros», numeral 19.3.4.11 registros de las transfusiones en el expediente clínico del receptor, lo que muestra una validez oficial por el Sistema Nacional de Salud.

Análisis de datos: el análisis estadístico se sometió al procesador de datos estadísticos SPSS V24 para el manejo de la información obtenida mediante gráficos para la captura de información. Para las variables cualitativas se utilizaron frecuencias simples y porcentajes, para las cuantitativas medidas de tendencia central y de dispersión.

\section{RESULTADOS}

En el periodo de enero de 2016 a abril de 2018 se registraron un total de 24,470 eventos transfusionales, de los cuales se encuentra un promedio de 890.11 transfusiones por año. En la Tabla 7 se muestran desglosadas por año, en el año 2017 hubo el menor registro de eventos transfusionales con un promedio de 841 transfusiones.

En este sentido, el registro de signos vitales pre, trans y postransfusión en hoja de registro del proceso de transfusión proporcionado por enfermería se encuentra con cambios oscilantes entre los meses, mostrando un mayor porcentaje de 


\begin{tabular}{lccc}
\multicolumn{4}{c}{ Tabla 1: Medias de tendencia central y de desviación para el número de } \\
transfusiones por año que se realizan en el hospital.
\end{tabular}

$D E$ = desviación estándar.

Figura 1:

Porcentaje de cumplimiento del registro de signos vitales por enfermería en el pre, trans y postprocedimiento transfusional.

Datos proporcionados por el Banco de Sangre del Hospital General Regional No. 1 «Vicente Guerrero».

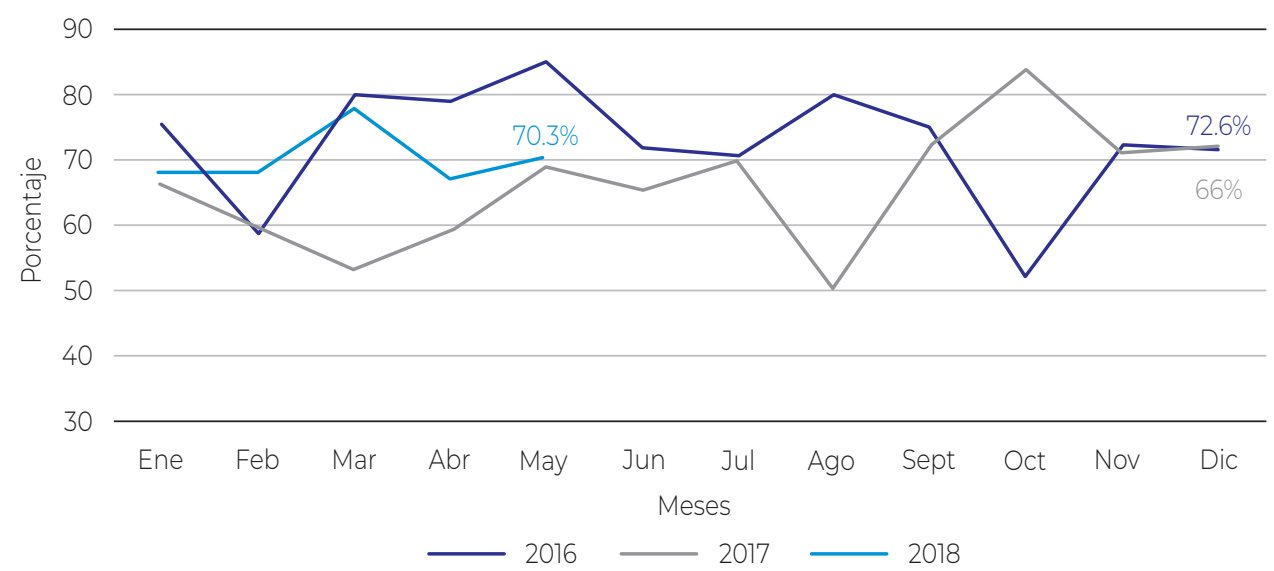

registro clínico para el año 2016 con un 72.6\%; por otro lado, se encuentra un contraste sumamente importante en el mes de octubre para el año 2017 en comparación con el año 2016, encontrando un 84 y $52 \%$ respectivamente. Los datos se muestran en la Figura 7.

De acuerdo con los resultados del tamaño de muestra, de los 237 eventos transfusionales, a los que se les realizó una evaluación exhaustiva de los reportes contenidos en el expediente clínico de manera aleatorizada, se encontró un mayor predominio de eventos transfusionales en el Servicio de Medicina Interna con un 56\%. Los datos se muestran en la Figura 2.

En relación con el porcentaje de cumplimiento del indicador de calidad en la atención de enfermería para la administración de hemoderivados, el de menor cumplimiento fue «en caso de reacciones adversas a la transfusión se registró su tipo y manejo, así como los procedimientos realizados» (24.8\%). Los datos se muestran en la Figura 3.

Por último, en relación con el porcentaje de cumplimiento a la NOM 253-SSA1-2012 mediante la evaluación de la documentación de hoja del registro del proceso de transfusión de hemoderivados fue de 9.87\%, quedando una falta de apego de $90.13 \%$, es decir, que no cumplen con los criterios que señala la norma. Los datos se muestran en la Figura 4.

\section{DISCUSIÓN}

De acuerdo con las Recomendaciones de la OMS, 18 "uso clínico de la sangre», se enfatiza la importancia de la educación y capacitación en el uso de este producto sanguíneo para todo el personal clínico y del Banco de Sangre involucrado en el proceso transfusional, este estudio demuestra cómo la 
sangre y productos sanguíneos son empleados de manera inapropiada en este hospital, comprometiendo los estándares de calidad y seguridad.

Bajo esta premisa, el estudio se realizó para identificar porcentaje de cumplimiento del indicador de calidad de la atención de enfermería, el apego NOM 253-SSA1-2012 transfusiones de hemoderivados en el Hospital General Regional No. 1 «Vicente Guerrero». En las 237 evaluaciones de las transfusiones sanguíneas realizadas se hace evidente una falta de cumplimiento al criterio de toma de signos vitales antes, durante y después

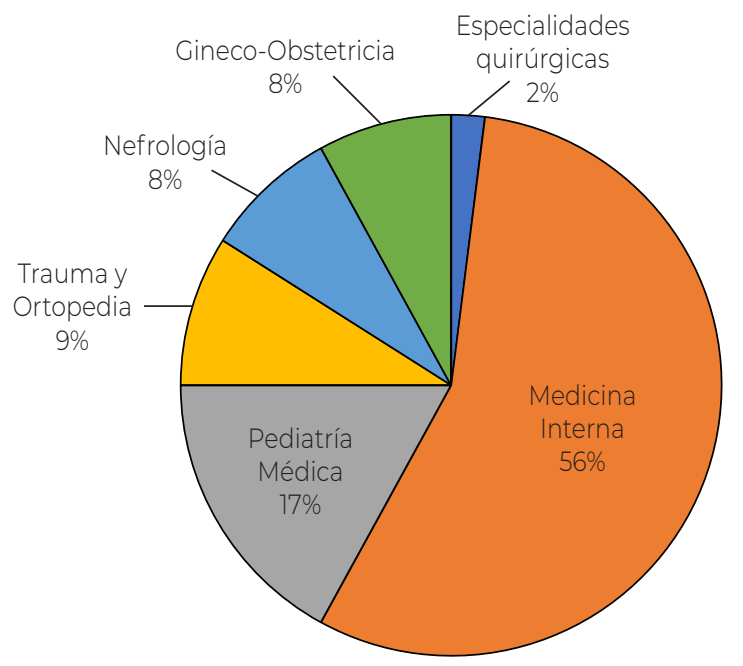

Figura 2: Servicios en los que se han realizado los eventos transfusionales. de la transfusión sanguínea, con un 67.95\% de incumplimiento.

Estos datos demuestran bajo desempeño y falta de responsabilidad del profesional enfermero en la documentación de su actuar en la hemotransfusión, con lo cual podemos inferir que es necesario determinar el nivel de preparación profesional y actualización y/o capacitación continua con los diferentes procedimientos del proceso de la atención en enfermería, principalmente entre el personal de mayor edad que muestra desinterés por la actualización y continúa con prácticas rutinarias de usos y costumbres; personal de nuevo ingreso que desconoce los criterios de la NOM 253 para el proceso de transfusión de hemoderivados.

Al comparar los resultados del presente estudio con los de Morales-Olarte E y colaboradores ${ }^{13}$ encontramos discrepancias importantes en relación con la carencia de los registros de acuerdo con lo establecido en la NOM 253, con una diferencia del 40.13\% entre ambos, siendo superior el incumplimiento en el presente estudio que en el realizado hace casi 20 años, esto podría justificarse por el cambio generacional de la plantilla de enfermeras, donde el 50\% de enfermeras generales son de nuevo ingreso a la institución, quizá por ello, se puede inferir la falta de vocación de servicio e identidad profesional al insertarse al sistema de salud.

De acuerdo con Flores-Torrecillas R. y su equi$\mathrm{po}^{14}$ se encontraron algunas coincidencias, las enfermeras revisan los datos de los pacientes, solicitud
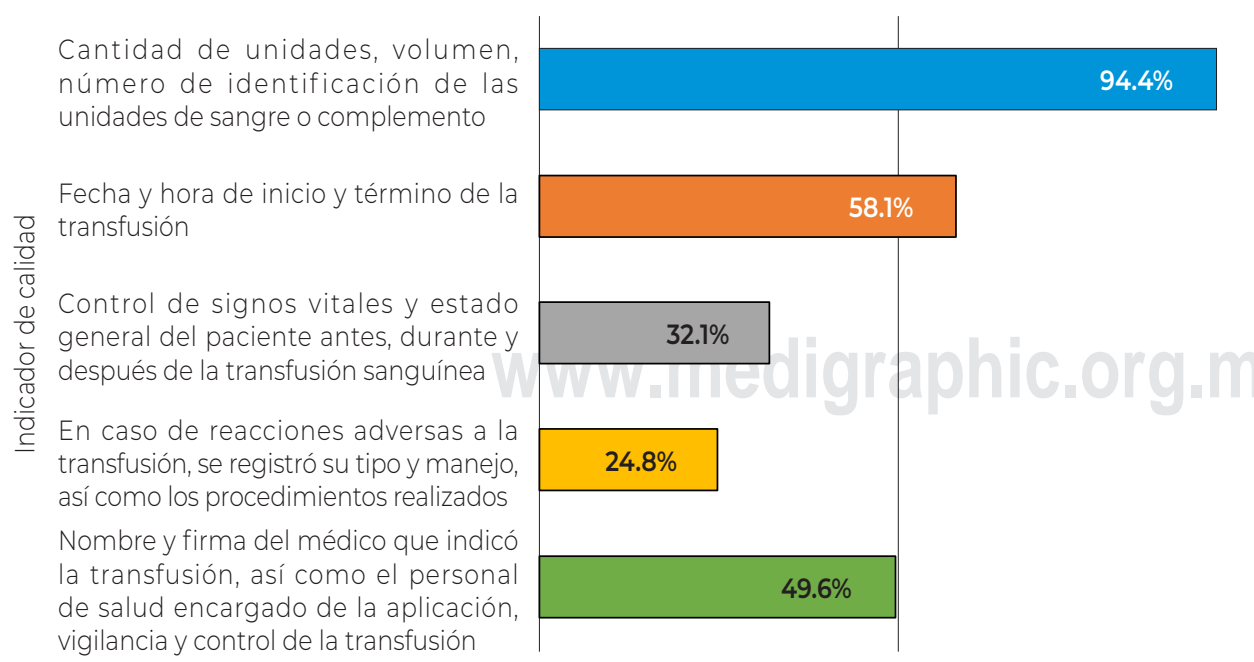

Porcentaje de cumplimiento*

\section{Figura 3:}

Porcentaje de cumplimiento del indicador de calidad de atención de enfermería del IMSS NOM 253-SSA1-2012 «transfusión de hemoderivados» por criterios. * Porcentaje de cumplimiento total del indicador de calidad 51.8\%. 


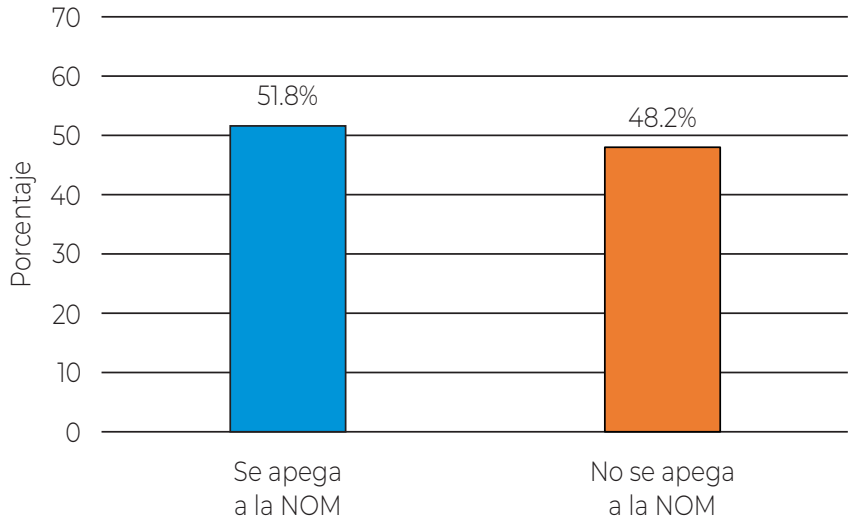

Figura 4: Porcentaje de apego a la NOM 253-SSA2-2012 de transfusión de hemoderivados.

de productos sanguíneos en el 99.1\% de los casos, mientras que en el presente estudio un $94.44 \%$. No obstante, existe una diferencia importante en el registro de signos vitales, ya que al comparar dicho cumplimiento, encontramos un 90.9\% de Flores Torrecillas R. y un 32.05\% del presente estudio. Estas discrepancias podrían verse influenciadas por la diferencia de regiones en donde se han realizado los estudios, siendo el primero en la parte norte del país y el presente estudio en la parte sur, el cual puede verse afectado por el nivel educativo bajo del estado de Guerrero, lo que puede conllevar una supervisión ineficaz del proceso de la atención de enfermería, el profesionalismo del personal en prácticas seguras.

Por otra parte, al contrastar los resultados de este estudio con los de Velasco-Rodríguez R y su grupo ${ }^{15}$ también existen discrepancias en el cumplimiento del registro de signos vitales, no lo realizan antes y después de la transfusión con 18.7 y $29.6 \%$ respectivamente. En este punto son preocupantes los resultados del estudio realizado en el HGR No. 1 «Vicente Guerrero», ya que se identificó que el $67.9 \%$ de las trasfusiones no registra los signos vitales en los tres tiempos de toma, o dicho registro se encuentra incompleto.

Por último, este estudio refiere que el 51.8\% de las transfusiones realizadas cumple con el indicador de calidad en la atención de enfermería para la administración de hemoderivados, no obstante, comparado con el nivel de cumplimiento de la documentación de 237 expedientes transfusionales sólo el 51.8\% se apega al registro de proceso de transfusiones sanguíneas de acuerdo con la NOM 253-SSA1-2012. Este incumplimiento y falta de apego a la norma, así como divergencias entre los resultados de otros estudios, pueden corresponder a la objetividad de la evaluación de indicadores, las cuales distan entre profesionales, lo que requiere de una experticia y preparación o conocimiento en el tema. Otro punto que puede influir en ello es el responsable que asume su rol de supervisor, el cual realiza sus actividades de prevención y gestión de riesgos y problemas, así como dimensionar la magnitud, trascendencia y vulnerabilidad de dicho procedimiento, pues aún no consideran esta práctica de alto riesgo porque no han trascendido los eventos adversos ocurridos.

Bajo esta premisa, el papel del gerente de enfermería debe ser proactivo, a efectos de detectar los fallos en la atención e instrumentar planes de mejora, identificar patrones y tendencias de eventos adversos y cuasi fallas.

\section{CONCLUSIONES}

Los resultados del estudio son alarmantes, ya que muestran un incumplimiento elevado del indicador de calidad para la administración de hemoderivados, lo cual pone en riesgo la calidad del proceso de transfusión y por ende la seguridad del paciente. Por ello, se hace necesario implementar acciones de mejora que garanticen una atención de calidad, para ello se necesitan designar criterios administrativos orientados a efectuar planes estratégicos de mejora continua como capacitación, difundiendo la guía de práctica clínica e intervenciones de enfermería para la seguridad en el manejo de la terapia transfusional.

\section{REFERENCIAS}

Organización Mundial de la Salud (OMS). Carta de Ottawa para la promoción de la salud. Primera Conferencia Internacional sobre la Promoción de la Salud. 1986.

2. Cuevas-Budhart MA, González-Martínez BR, Ayala-Torres M, Castro-Narváez X, Leyva-Alvarado C, Bahena-Acevedo DL et al. Incumplimiento de los indicadores de calidad de estructura en unidades de la Dirección General de Salud Municipal en Acapulco, Guerrero. Rev CONAMED. 2018;23 (1): 12-16. 
3. Martínez-Matheus M, Rodríguez-lbagué LF, CárdenasLlano CL. Indicadores de gestión en los Programas Académicos de Campo (PAC) del programa de fisioterapia de la Universidad del Rosario para el periodo 2004-2007. Rev Cienc Salud. 2010; 8 (3): 7-18.

4. Álvarez-Bolaños E, Ríos-Barreda CP, Cuevas-Budhart MA. Cumplimiento de las dimensiones del indicador Correlación de indicaciones médicas con acciones de enfermería. Rev Enferm Inst Mex Seguro Soc. 2018; 26 (1): 23-28.

5. Cuevas-Budhart MA, Martínez BR, Bolaños EA, Barrera MJ, De Almeida Souza A, Conzález-Jurado MA et al. Diseño y validación de un nuevo registro clínico de enfermería, para la continuidad de los cuidados y seguridad del paciente en hemodiálisis. Enferm Nefrol. 2019; 22 (2): 168-1675.

6. Caamaño C, Ramón Martínez J, Alonso M, Hernández A, Martínez-Renedo E, Sainz A. Indicadores de calidad de los cuidados de enfermería hospitalarios. Rev Calidad Asistencial. 2006; 21 (3): 143-149.

7. NORMA Oficial Mexicana NOM-253-SSA1-2012, Para la disposición de sangre humana y sus componentes con fines terapéuticos. Centro Nacional de la Transfusión Sanguínea.[Internet]. [citado 08 de julio de 2019]. Disponible en: http://www.dof.gob.mx/normasOficiales/4917/salud3a/ salud3a.html

8. Narro Robles JR. 4to Informe de labores. México; 2015.

9. Meybohm P, Richards T, Isbister J, Hofmann A, Shander A, Goodnough LT et al. Patient blood management bundles to facilitate implementation. Transfus Med Rev. 2017; 31 (1): 62-71.

10. Rojo-Medina J. Blood supply system in Mexico. ISBT Sci Ser [Internet]. 2012 [cited 2019 Jul 8]; 7 (1): 244-250. Available from: http://doi.wiley.com/10.1171/j.1751-2824.2012.01604.x
11. Cortés Fadrique C, del Trigo Méndez P, Veiga FR, Sánchez Bermejo R, Rincón Fraile B, Fernández Centeno E. En torno a los hemoderivados. Enfermería Global. 2014; 14 (1):23-37.

12. Cuevas Budhart MA, Saucedo García RP, García Larumbe JA, Álvarez Bolaños E, Pacheco del Cerro E, Meneses Monroy A et al. Factores asociados al desarrollo de eventos adversos en pacientes con hemodiálisis en Guerrero, México. Enferm Nefrol. 2019; 22 (1): 42-50.

13. Morales Olarte E, Reyes Morales H, Mercedes J, Canto F, Martínez García MC. Problemas técnicos de enfermería durante la transfusión de componentes sanguíneos. Rev Enferm IMSS. 1999; 7 (1): 37-41.

14. Flores-Torrecillas R, Carballo-Monreal MR, ÁlvarezVillaseñor AS, Valdez-Márquez ML, González-Ojeda A, Fuentes-Orozco C. Manejo y administración de hemoderivados por personal de enfermería en un hospital de segundo nivel. Enferm Univ. 2014; 11 (3): 94-100.

15. Velasco-Rodríguez R, Osorio-Cruz AD, Peguero-Ortiz RE, Mora-Brambila AB. Cumplimiento en el manejo de hemoderivados por personal de enfermería en un hospital de México. Rev Enferm IMSS. 2013; 21 (1): 15-21.

16. Padovani Cantón M. Indicadores de resultado para la evaluación de la calidad en Unidades de Cuidados Intensivos. Rev Ciencias Médicas. 2014; 18 (3): 441-452.

17. División de Mejora a la Gestión de los Servicios de Salud (DMCSS). Coordinación de Políticas en Salud, Unidad de Educación, Investigación y Políticas en Salud, Instituto Mexicano del Seguro Social. México. Salud Pública de México; 2015.

18. Organización Mundial de la Salud. El uso clínico de la sangre en medicina, obstetricia, pediatría y neonatología, cirugía y anestesia, trauma y quemaduras. Cinebra : Organización Mundial de la Salud. 2002. 